\title{
Biopsy Specimen Radiography
}

National Cancer Institute

\section{Source}

National Cancer Institute. Biopsy Specimen Radiography. NCI Thesaurus. Code C137813.

Radiog raphic examination of a specimen obtained by biopsy or surgical procedure. 